\title{
Finite element analysis of temporary pre-stress system of precast segmental box girder bridge
}

\author{
Wang Yahui ${ }^{1}$, Yang Zheng ${ }^{1}$, Kang Jiangwei ${ }^{1}$ and Li Zhongming ${ }^{2}$ \\ 1. School of Human Settlements and Construction Engineering, Xi'an Jiao tong University, Xi' an, 710048, Shanxi, China \\ 2. China Construction Seven Municipal Engineering Co., Ltd, Zhengzhou, 4500444, Henan, China
}

\begin{abstract}
The precast segmental bridge has the advantages of fast construction speed, good economic performance, and small impact on the surrounding environment. In order to study the role of the temporary pre-stressing system in the cantilever assembly process of the precast segment box girder bridge, ABAQUS was used to simulate the assembly process of the standard section of a viaduct. Studying the compressive stress and relative sliding of the joint section under the action of the self-weight and temporary pre-stress, the reasonable number and spatial arrangement of the tension pedestal are determined. The research results show that too few roof pedestals will cause the large area decompression state and too much will cause the construction period to be longer. When the roof symmetrical pedestal is located on the inner side of the web, it will obviously increase the area of the zero-compression stress at the end of the top slab. When the distance between the pedestal and the center of the web is $0.12 \mathrm{~L}$, the full section meets the minimum consolidation pressure requirement of the glued joint. The reasonable number of tension pedestals and their spatial arrangement will provide specific guidance and reference to assembling construction of actual precast segment box girder in the future.
\end{abstract}

\section{Introduction}

Precast segmented bridges have the advantages of fast construction speed, good economic performance, and small impact on the surrounding environment ${ }^{[1]}$. A large number of medium and long span bridges have gradually adopted this construction technology to replace the castin-place method. During the cantilever precast assembly process of the precast segmental box girder bridge, according to the design and site construction environment, the test assembly of the box girder segments should be carried out first, and then epoxy resin glue ${ }^{[2]}$ should be applied to the joints' section of the segments, and finally temporary pre-stress which use high-strength rebar is applied in accordance with the design load requirements ${ }^{[3]}$. During the cantilever assembly of the precast segment box girder bridge, the temporary pre-stress system can accurately locate the spatial position of the precast box girder according to the design requirements and ensure the safety and stability of the box girder structure before permanent tensioning. The pre-tightening force provided by the finish-rolled rebar ensured that the epoxy resin at the joints of the segments meets the minimum consolidation compressive stress of $0.3 \mathrm{Mpa}^{[4]}$.

The temporary pre-stressing system plays a vital role in the safety and stability of the cantilever assembly structure during the segment assembly process ${ }^{[5]}$, and it is necessary to conduct a detailed study on the temporary pre-stressing system. Ai Z L from Southeast University discussed the modeling method of segmented pre-stressed concrete bridges $^{[6]}$, calculated and analyzed the cantilever assembly construction process, and then proposed a reasonable temporary pre-stressed tension sequence. Yu M and Zou $\mathrm{H}$ of the China Communications Second Navigation Bureau introduced a new type of temporary tensioning system - shear cone tensioning system, which was successfully applied to the Hong Kong-ZhuhaiMacao Bridge ${ }^{[7]}$. Deng $G$ studied the temporary prestressing system of a viaduct, using the forward installation method to numerically simulate the stress status and temporary pre-stress effect of the structure during the construction process ${ }^{[8]}$.

At present, researches on temporary pre-stressing systems at home and abroad have focused on simple linear analysis and new temporary pre-stressed pedestal invented for the convenience of construction. However, the stress of the box girder section is complicated due to the shear lag effect. And there are few researches are about the influence of different number and space layout of tension pedestal on the structure force and deformation performance in the construction stage. The purpose of this article is to analyze the stress and deformation performance of the glue joint section during the assembly process of the precast segment box girder, and then determine the scientific and reasonable pre-stress tension system, that is, the reasonable number of tension pedestals and their spatial arrangement, so as to provide specific guidance and reference to assembling construction of actual precast segment box girder. 


\section{Temporary pre-stressed system and finite element model}

\subsection{Temporary pre-stress system}

Taking the cantilever assembly process of a standard viaduct segment as the research object. In the actual cantilever assembly of box girder, after the segment girder is accurately aligned, the temporary pre-stress is immediately tensioned, and the roof is arranged with three steel structure tensioning pedestals, 6 Ф24 precision rolled rebar. A tensioning pedestal is arranged on the bottom

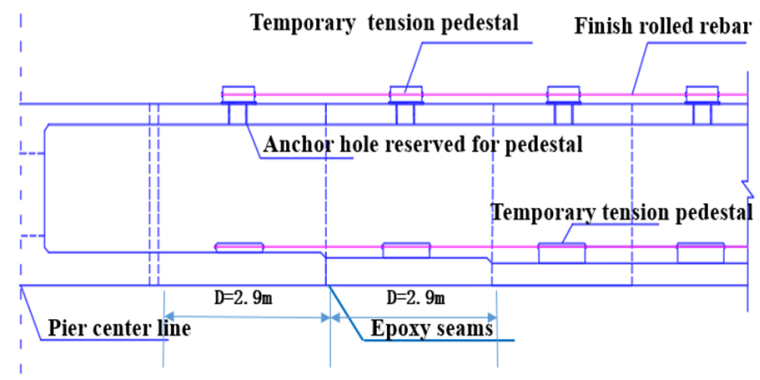

plate, and $\Phi 32$ fine-rolled high-strength rebar are used as a temporary pre-stressing system. The layout of the temporary pre-stressing tensioning pedestal is shown in Fig.1. Before temporary pre-stressing, a detachable steel member is installed on the top plate as the tensioning system. After the beam body is assembled, M55 microexpansion cement slurry should be used to block the temporary pre-stressed pedestal anchoring reserved holes on the top plate. After the temporary pre-stress is used, it can be removed from the closing section. The pre-stress tensioning adopts 2 sets of $60 \mathrm{t}$ through-core Hydraulic Press which are symmetrical on the left and right, and proceed in order from the inside to the outside ${ }^{[9]}$

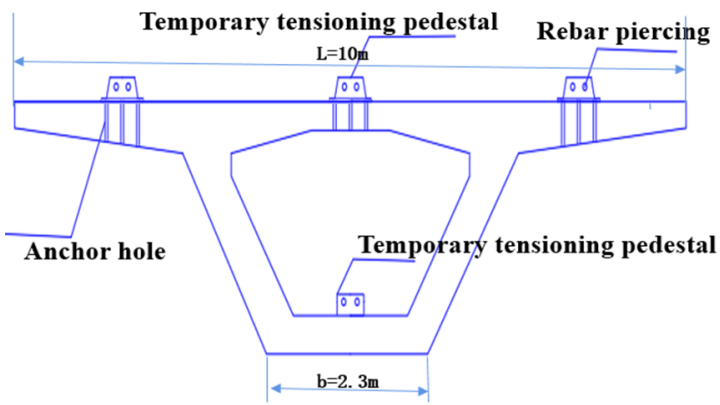

Fig. 1. Schematic diagram of the box girder and tension pedestal

Now select a representative standard section of box girder with width $\mathrm{L}=10 \mathrm{~m}$ and length $\mathrm{D}=2.9 \mathrm{~m}$ to study the compressive stress and the relative sliding deformation of the joint between the spliced section $n$ and the $n+1$ section to be spliced ${ }^{[10]}$. The design temporary pre-stress of the top plate is $F_{1}=1800 \mathrm{KN}$, and the design temporary prestress of the bottom plate is $F_{2}=900 K N$. The section to be assembled is consolidated on previously assembled section through temporary pre-stress and epoxy resin, the relevant mechanical model is shown in Fig.2. Now fixing the number and location of the bottom plate tensioning pedestals and keeping the design temporary pre-stress unchanged, the finite element analysis is carried out on the temporary pre-stressing system in the cantilever construction of box girder bridge. We can compare the advantages and disadvantages of the different number of tension pedestal and their space layout on the top plate

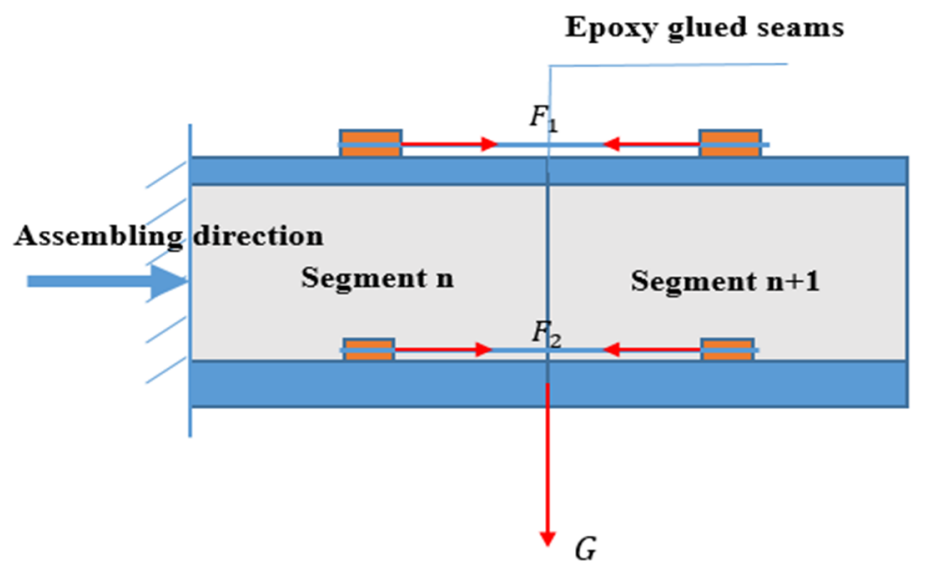

Fig. 2. Schematic diagram of mechanical model

\subsection{Finite element model}

\subsubsection{Material properties}

Because the box girder in the cantilever assembly stage is in the elastic stage under the action of the structure's own weight and the temporary pre-tightening force, only setting the elastic parameters can meet the calculation requirements. The relevant material performance parameters of the precast segment box girder bridge of this project are shown in Table 1, E stands for elastic modulus, $v$ stands for Poisson's ratio, $\rho$ represents material density. 
Table 1. Material parameters of box girder

\begin{tabular}{ccccc}
\hline Structural member & Material & E & $v$ & $\rho\left(\mathrm{Kg} / \mathrm{m}^{3}\right)$ \\
\hline Concrete box girder & C60 & $3.6 \times 10^{10}$ & 0.3 & 2450 \\
\hline Tension steel pedestal & Q235 & $2.0 \times 10^{11}$ & 0.25 & 7850 \\
\hline
\end{tabular}

\subsubsection{Contact, restraint, and load properties}

Contact relationship: the surface between the bottom surface of the tensioning pedestal and the top of the box girder adopt tie constraints, as for the glue joint section which connecting the spliced section $\mathrm{n}$ and the $\mathrm{n}+1$ section to be spliced, the normal behavior of the epoxy joint is defined as hard contact, the friction formula in the tangential behavior is defined as a penalty function, and the friction coefficient is 0.6 . The load only considers the self-weight and temporary pre-stress during the cantilever assembly stage. As shown in Figure 2, the boundary condition of the end of the nth segment is completely fixed, constraining the displacement and bending in the three directions of $\mathrm{x}, \mathrm{y}$, and $\mathrm{z}$. Under the self-weight and temporary pre-stress, the stress and deformation of the spliced $n$th section and the $n+1$ th section to be spliced are studied $^{[11]}$. The finite element model is shown in Fig. 3.

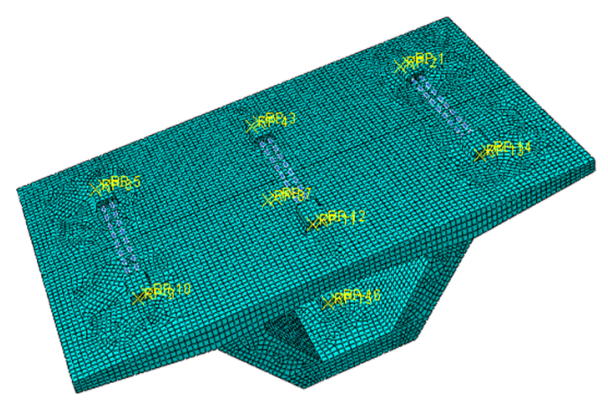

Fig. 3. Schematic diagram of finite element model

\subsubsection{Grid and element properties}

The finite element model of the space box girder and the tension pedestal adopts the C3D8R element (threedimensional stress eight-node linear hexahedral element), adopts reduced integration, hourglass control. The mesh size is $0.1 \mathrm{~m}$. Because only the pre-stress effect on the box girder is considered and the stress and strain of the finished rolled rebar are not considered, it is reasonable to use connector connection element to simulate the pre-stress effect.

\section{The number influence of temporary top tensioning pedestals}

In this paper, finite element analysis and calculation are used to study the stress performance and deformation capacity of box girder structures with different numbers of top pedestals $(\mathrm{m}=1,2,3,4,5)$ under self-weight and prestress load. The pedestals are on the top plane. The layout is evenly arranged based on the three pedestals designed on the top of the box girder, the relevant layout is shown in Fig.4.

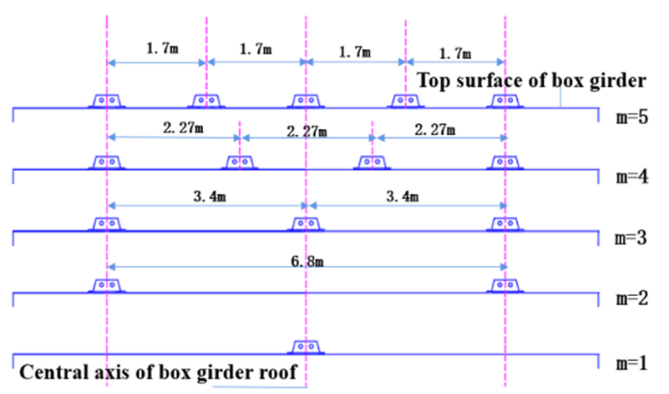

Fig. 4. Schematic diagram of top pedestal' number changes

Selecting representative upper and lower edge compressive stress of the epoxy resin adhesive joint crosssection for analysis and comparison. From Fig.5(a), it can be found that the number $m$ of roof tension pedestals has a great influence on the size and distribution of the compressive stress of the top slab section. When the number of pedestals is 1 , due to the Saint-Venant effect, the temporary pre-stress effect has a limited range, there is compressive stress only within $0.9 \mathrm{~m}$ near the central axis of the box girder top plate, the rest area is close to the depressurized state, which is very unfavorable for the consolidation of epoxy resin. As m gradually increases, the zero-compressive stress state of the cross-section gradually disappears. It can be seen from Fig. 5(b) that if $\mathrm{m}$ is too small, the compressive stress on both sides of the bottom plate section will be too small. When $m=3$, most of the cross-sections of the bottom and top plates have already met the minimum consolidation compressive stress requirements of epoxy resin. Increasing the number of tension pedestals $\mathrm{m}$, the maximum compressive stress at the middle of bottom plate will gradually increase. The local maximum compressive stress is reached in the middle of the bottom or the middle of the top, which is caused by the shear lag effect ${ }^{[11]}$. Continuing to increase the steel component tensioning pedestal can continue to enhance the section mechanical properties, but it will undoubtedly increase the construction work and delay the construction period.

Fig. 5(c) shows the deflection of the central axis of the top plate of the cantilever assembled box girder structure under its own weight. When relative position of the horizontal axis is 0 , the corresponding position is the glue joint. It can be clearly seen that there is a relative sliding phenomenon at the two-segment glued joint. As the number of pedestals $\mathrm{m}$ increases, the relative sliding distance of the joint gradually decreases. When $\mathrm{m}=5$, the 
relative sliding is $0.095 \mathrm{~mm}$, when $\mathrm{m}=1$, the relative sliding is $0.332 \mathrm{~mm}$. After the number of pedestals is greater than 3 , continuing to increase the number of pedestals has little effect on improving the dislocation of the joints. For the $\mathrm{L}=10 \mathrm{~m}$ wide standard box, reasonable number of tensioning pedestals is 3 to ensure the integrity and stability of the structure.

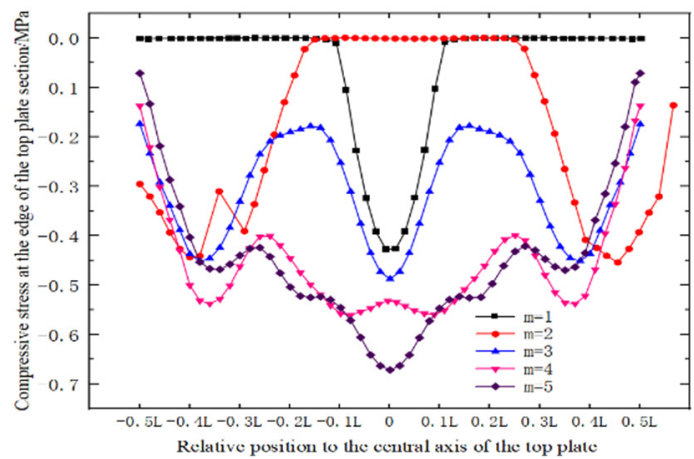

(a) Compressive stress of the upper edge of the roof section

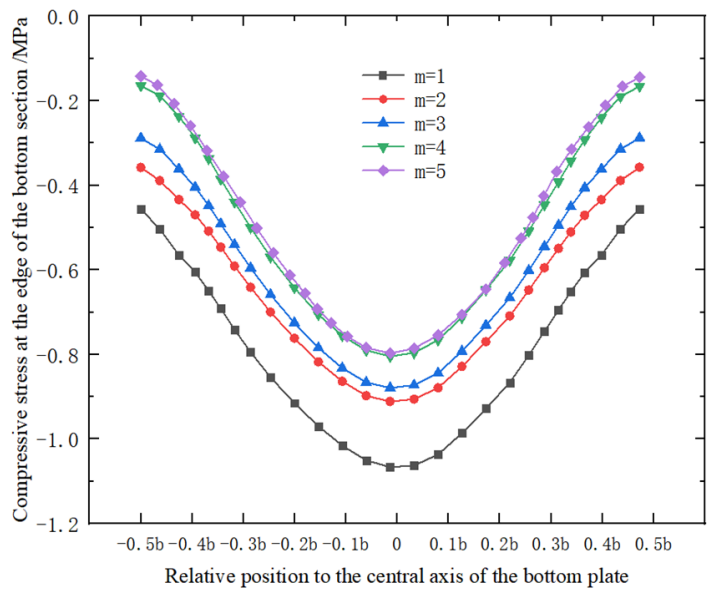

(b) Compressive stress of bottom edge of bottom plate section

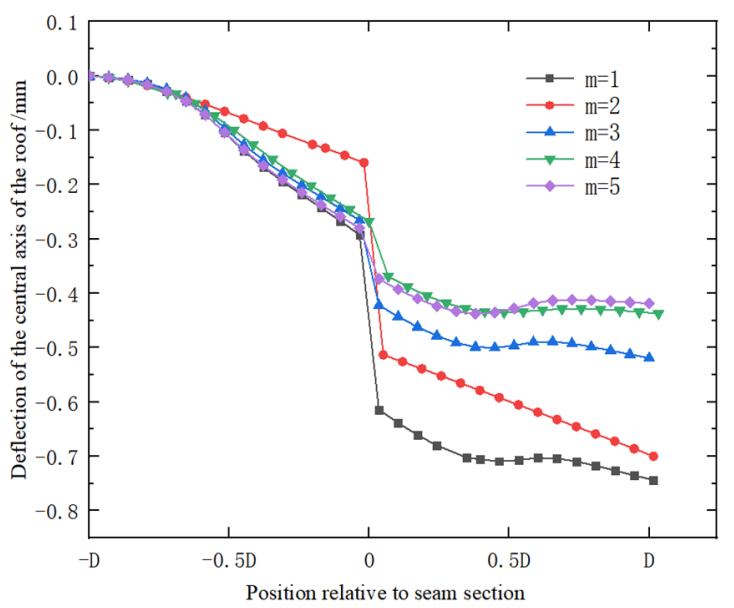

(c) Deflection of central axis of box girder roof

Fig. 5. The top pedestals number's effect on the top and bottom plate compressive stress and deflection

\section{Tensioning pedestals' space layout}

In order to ensure the safety and economic benefits of the box girder assembly process, it is advisable to adopt the design of three-tensioned top plate for the $\mathrm{L}=10 \mathrm{~m}$ wide standard section box girder. However, the rational arrangement of the tensioning pedestal on the top plate is related to whether the cantilever assembled box girder structure has excellent stress and deformation capabilities. The current construction adopts the spatial arrangement mode of three-tensioned roof pedestal. We can see that in Fig.6. Now we fix the pedestal of the central axis of the top plate and respectively change the lateral distance 1 , which is between the symmetrically arranged pedestals and the central axis of the web, then studying the situation when $\mathrm{l}=-0.04 \mathrm{~L}, 0 \mathrm{~L}, 0.04 \mathrm{~L}, 0.08 \mathrm{~L}, 0.12 \mathrm{~L}, 0.16 \mathrm{~L}$ (negative values represent symmetrical pedestals which is located on the inner side of the web), the numerical simulation analysis results are as follows:

As shown in Fig.7(a), it can be seen that changing 1 has great influence on the compressive stress of the top plate section. When the value of 1 is small, due to the SaintVenant effect, the maximum compressive stress in the middle of the upper edge of the roof will be larger, and the compressive stress will gradually increase from the sidewalk on both sides to the central axis, and the area of the zero compressive stress zone at the flanges on both sides will be larger, it is not conducive to the formation of the strength of the adhesive joint. When the distance between the symmetrical pedestal and the center of the web is $0.12 \mathrm{~L}$ upwards, the zero-compression stress zone at the end of the top plate disappears, and the basic full section meets the minimum consolidation pressure of the glued joint. If 1 continues to increase, the compressive stress at the junction of the web and the roof will gradually become smaller, even lower than the consolidation pressure.

It can be seen from Fig.7 (b) that after the number of top tensioning pedestals is determined, the symmetrical pedestal is located near the central axis of the web $(1=-$ $0.04 \mathrm{~L}, 0,0.04 \mathrm{~L}$ ), the structure will cause the end to warp due to excessive restraint. When the distance 1 is equal to $0.12 \mathrm{~L}$, the end of the box girder is approximately straight without warping, which is conducive to the assembly of the next section. After the number of top pedestals is determined, slightly changing the distance between the symmetrical pedestal and the central axis of the web has little effect on the deflection of the structure and the relative sliding of the joint.

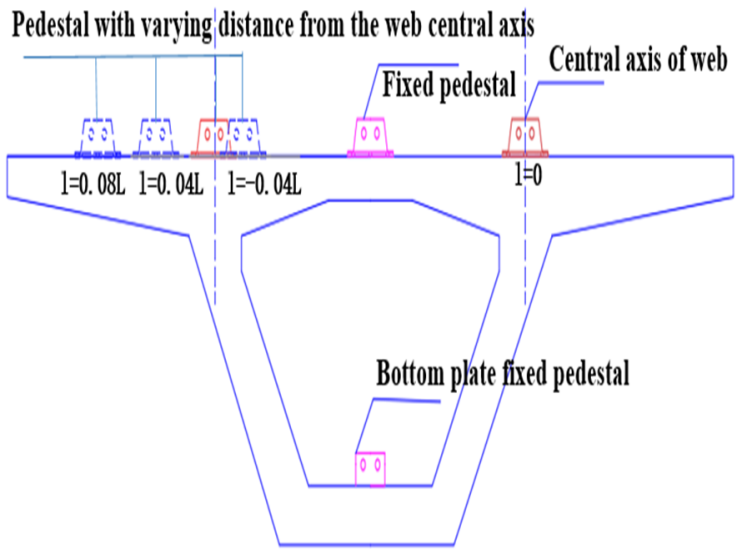

Fig. 6. Schematic diagram of the change of the distance 1 


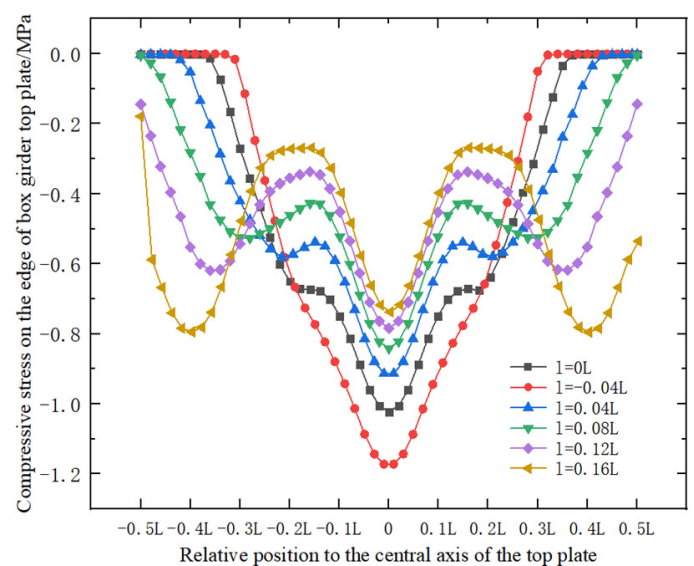

(a) Compressive stress of the upper edge of the roof section

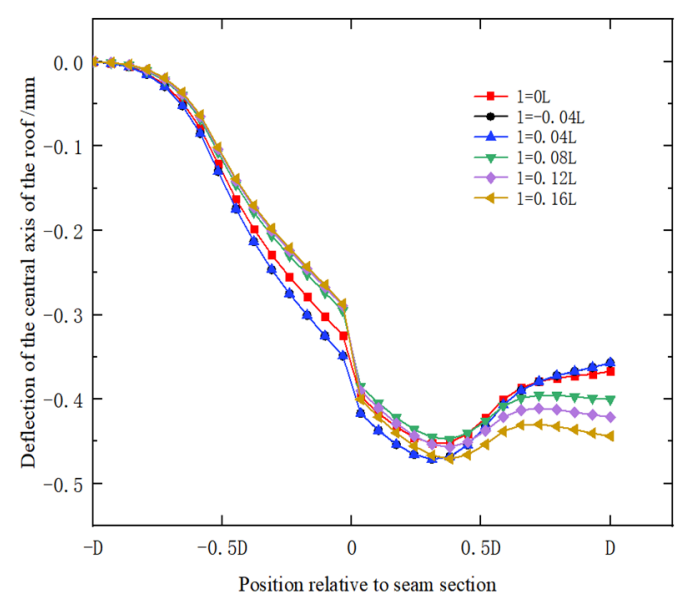

(b) Deflection of central axis of box girder roof

Fig. 7. The influence of the distance 1 on on the top plate compressive stress and deflection

\section{Conclusion}

For the prefabricated segment box girder section of specific geometric dimensions, we can determine a reasonable number of pedestals and spatial arrangement, which can improve the overall stress performance of box girder during cantilever assembly and ensure the normal consolidation of the epoxy resin at the joints.

(1) Too little number of tensioning pedestals will result in insufficient local compressive stress of joints or even partial decompression. Excessive number of tensioning pedestals will lead to a longer construction period. By comprehensive considerations, the top 3 tension pedestal basically meets the requirements.

(2) When the top plate symmetrical tension pedestal is located on the inner side of the web, the maximum compressive stress in the middle of the top plate edge will increase, this kind of pedestal layout will obviously increase the area of the zero compressive stress. When 1 is equal to $0.12 \mathrm{~L}$, the zero-compression stress zone disappears, and the full section meets the minimum consolidation pressure of the glued joint. If 1 continues to increase, the compressive stress at the junction of the web and the top plate will gradually decrease, even lower than the colloidal consolidation pressure. Considering comprehensively, the reasonable horizontal arrangement should be that the distance 1 between the symmetrical top tensioning pedestal and the central axis of the web is near $0.12 \mathrm{~L}$.

\section{References}

1. Arockiasamy M, Reddy D V, Sivakumar M, et al. Fatigue Loading and Temperature Distribution in Single Cell Segmental Box Bridges [J]. Practice Periodical on Structural Design \& Construction, (2008), 13(3): 118-27.

2. Ma S J, Ying S, Dai Z P. Study on Construction Technology of Precast Segmental Bridge [J]. Municipal Technology, (2006), 024(003): 148-52.

3. Huang Y, Wu X. Study on Prefabrication and Installation Technology of Broad Width Box Girder Segment for Approach Bridge of Quanzhou Bay Bridge [J]. Construction Technology, (2015).

4. Liu H L, Xu D Z. Humen Bridge across $62.5 \mathrm{~m}$ Segmental PC continuous rigid frame bridge design department [J]. Heilongjiang Transportation Technology, (2015), 000(005): 66-7.

5. Li X B. Study on the mechanical characteristics of externally prestressed concrete box girder assembled with precast segments [D]; Academy of Railway Sciences, (2003).

6. Ai Z L. Research on construction technology of shortline matching prefabrication and cantilever assembly of box girder [D]; Southeast University, (2006).

7. Miao Y U, Zou H. Application of Novel Temporary Tension System in Short-Line Matching Cantilever Assembly Bridge [J]. (2017).

8. Deng G. Design of Temporary Prestress in Cantilever Construction of Precast Segmental Beams [J]. Transportation Science \& Technology, (2019), 000(003): 24-5,9.

9. Zhang Q C, Wang B B. Intelligent complete construction technology of precast segment beam prestress system [J]. Engineering Quality, (2020), 038(0z1): 32-4,40.

10. Zhou J. Short-line Prefabrication and Cantilever Assembly Technique of Sutong Bridge $75 \mathrm{~m}$ Continuous Pre-stressed Concrete Girder [J]. Modern Transportation Technology, (2007)..

11. Chen D. On shear lag effect analysis of prestressed box girder at cantilever construction stage [J]. Shanxi Architecture, (2012). 